\title{
Pain and rehabilitation after total hip arthroplasty are approach dependent: a multisurgeon, single-center, prospective cohort study
}

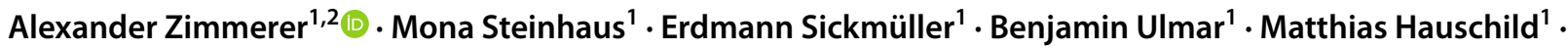 \\ Wolfgang Miehlke ${ }^{1} \cdot$ Stefan Kinkel ${ }^{1}$
}

Received: 7 August 2020 / Accepted: 23 April 2021 / Published online: 8 May 2021

(c) The Author(s) 2021

\begin{abstract}
Purpose The aim of this study was to assess perioperative pain and mobilization after total hip arthroplasty (THA) using three different surgical approaches.

Methods This was a multisurgeon, prospective, single-center cohort study. A total of 188 patients who underwent hip arthroplasty (THA) between February 2019 and April 2019 were analyzed according to the surgical approach used (direct anterior, lateral, and posterior approach). Outcome parameters were the daily walking distance during the inpatient stay, the pain level according to the visual analog scale (VAS) at rest and motion during the inpatient stay and at 6-week follow-up and the modified Harris Hips Score (mHHS) preoperatively and at 6 weeks.

Results The walking distance within the groups increased significantly during the inpatient stay $(p<0.001)$. The DAA and posterior approach patients had a significantly longer walking distance than the lateral approach patients on the third postoperative day (DAA vs. lateral, $p=0.02$; posterior vs. lateral $3, p=0.03$ ). DAA and posterior approach patients reported significantly less pain during motion on the third postoperative day and at 6-week follow-up than the lateral approach patients (3 postoperative day: DAA vs. lateral, $p=0.011$; posterior vs. lateral, $p=0.04 ; 6$ weeks control: DAA vs. lateral, $p=0.001$; Posterior vs. lateral $3, p=0.005$ ). The mHHS demonstrated significant improvement within each group. However, lateral approach patients reported significantly less improvement than the DAA and posterior approach patients (DAA vs. lateral, $p=0.007$; posterior vs. lateral, $p=0.021$ ).

Conclusion This study analyzed perioperative pain progression and short-term rehabilitation after THA according to the different surgical approaches. Direct anterior and posterior approaches have shown comparable improvements in pain, walking distance, and mHHS. Whether this effect persists over a longer period of time must be clarified in future studies.
\end{abstract}

Study design Prospective cohort study, level of evidence, 2.

Keywords Total hip replacement $\cdot$ Rehabilitation $\cdot$ PROM $\cdot$ Anterior approach $\cdot$ Lateral approach $\cdot$ Posterior approach

This study was approved by IRB/Ethikkommission

Landesärztekammer Baden-Württemberg, Germany.

Alexander Zimmerer

zimmerer@sportklinik.de

1 ARCUS Sportklinik Pforzheim, Rastatterstr. 17-19, 75179 Pforzheim, Germany

2 Department of Orthopedics and Orthopedic Surgery, University Medicine Greifswald, Ferdinand-SauerbruchStraße, 17475 Greifswald, Germany

\section{Background}

Coxarthrosis is a common disease of the hip joint and it is typically treated by total hip arthroplasty (THA) if conservative therapy is not successful. The majority of patients undergoing THA experience pain relief, improved mobility, enhanced functionality and the restoration of the quality of life [1, 2]. Moreover, several studies have shown improved patient-reported outcome measures (PROMs) following THA [3, 4]. Nevertheless, a small minority of THA patients continue to suffer from symptoms, mostly pain, that prevent their return to full function and activity. Possible underlying causes include fixation failure, instability, and soft tissue damage, related to the trauma of the surgical procedure. 
The latter seems to have a major influence on the short-term results due to soft tissue damage [5-7].

The three most common methods used for THA are the anterior, lateral, and posterior approaches [8]. However, there is still no consensus on the effect of the surgical approach on postoperative pain. In recent years, many studies have compared two of these approaches (anterior vs. posterior, anterior vs. lateral, posterior vs. lateral) [5, 9-15]. In these studies, the anterior approach appears to be superior to the other approaches in terms of postoperative pain and short-term functional outcome. To the best of our knowledge, only a few studies have investigated and compared all three approaches against each other according to their effect on postoperative pain after THA $[8,16,17]$. Therefore, the aim of this study was to assess perioperative pain and mobilization after THA in relation to the three different surgical approaches. We hypothesized that DAA patients will report less pain and mobilize more quickly than patients who receive the lateral or posterior approach.

\section{Methods}

The present study comprises a consecutive series of 188 patients following cementless total hip arthroplasty performed in a single-center, multisurgeon series (7 surgeons) between February 2019 and April 2019. Patients were evaluated prospectively over the study period. The Corail Pinnacle Hip System (DePuy Synthes, Warsaw, IN) was used in all patients. Three groups were formed according to the approach used: Group 1, minimally invasive direct anterior approach (DAA); Group 2, transgluteal lateral approach; Group 3, piriformis muscle sparing posterior approach. Each surgeon performs THA with only one approach at high volumes ( $>250 /$ year). Thus, every patient of the respective surgeon was operated on using the same approach. The inclusion criteria consisted of primary cementless total hip arthroplasty and consent for general anesthesia. Exclusion criteria included revision surgery, the existence of chronic pain syndrome preoperatively or for the use of epidural anesthesia (spinal block) to eliminate the bias of prolonged analgesia due to epidural anesthesia.

A standardized pain management concept was applied for all patients: Preoperatively the patients received 90-mg etoricoxib, 50-mg pregabalin and 20-mg pantoprazole. During surgery, dexamethasone at a dosage of $0.1-0.2 \mathrm{mg} / \mathrm{kg}$, ondansetron $4 \mathrm{mg}$ and tranexamic acid $500 \mathrm{mg}$ were administered intravenously. A propofol bolus of $1.5-2.5 \mathrm{mg} / \mathrm{kg}$ was used intravenously to induce anesthesia. In addition, fentanyl $0.2 \mathrm{mg}$ and the muscle relaxant rocuronium $0.5-0.6$ $\mathrm{mg} / \mathrm{kg}$, which is monitored and controlled by relaxometry during surgery, were injected. Anesthesia was maintained by a continuous propofol infusion device. Postoperative oral-controlled analgesia was applied at the ward. The patients received $500 \mathrm{mg}$ of metamizole four times daily and $90 \mathrm{mg}$ of etoricoxib once daily as the standard. During the first 2 days, the patients received an additional 10-mg Targin in the morning and evening. During pain peaks, 10-mg Sevredol was administered every $4 \mathrm{~h}$ as needed.

Full weight-bearing was allowed in the immediate postoperative course. The initial mobilization of the patients was performed on the day of the operation as a standard, and patients also received physiotherapeutic treatment once a day from the day of the operation onwards. After discharge, pain-adapted full weight-bearing was further permitted. During the first 4 weeks, flexion over $90^{\circ}$ and terminal rotational and adduction movements were prohibited. Ossification prophylaxis was given during the first 2 weeks, and thrombosis prophylaxis was given during the first 4 weeks postoperatively. The postoperative rehabilitation protocol was the same for all surgeons.

As a matter of routine, patients were prospectively assessed preoperatively and at 6 weeks postoperatively using the modified Harris Hip Score (mHHS) [18, 19]. The pain level was evaluated using a visual analog scale (VAS) for pain (VAS $0=$ no pain; VAS $10=$ worst pain imaginable) and was recorded preoperatively and on each day of hospitalization for rest and motion. It was also recorded 6 weeks postoperatively. The time of the initial mobilization as well as the daily walking distance were documented during the inpatient stay. In addition, factors such as depression, chronic pain syndrome, and diabetes that have been identified in the past as negative prognostic factors [20-23] for postoperative outcomes were documented. All data were collected daily by one investigator (M.S).

The ethics commission of the Landesaerztekammer Baden-Wuerttemberg, Germany approved all procedures (F-2019-006), and the study was conducted in accordance with the Helsinki Declaration of 1975, as revised in 2008. The study was registered in the German Registry of Clinical Studies (DRKS) with the approval number DRKS00016519 (WHO Register). All participants provided written informed consent.

\section{Statistics}

$\mathrm{G}^{*}$ Power [24] (version 3.1.9.2, 2014) was used for the sample size calculation. Assuming 5\% as the acceptable margin of error, it was estimated that a total sample size of 180 patients would have $80 \%$ power in the study. Descriptive statistics for all continuous variables are reported as the means \pm standard deviations. Differences between preoperative and postoperative data were examined with a $t$ test and a Wilcoxon signed-rank test. Differences between the groups were tested using ANOVA test with a Bonferroni correction for multiple repeated comparisons. Categorical 
variables were reported using the count and percentage. To compare percentages between groups, Fisher's exact test was performed. Statistical analyses were conducted using SPSS statistical software (IBM SPSS Statistics for Windows, version 26.0.0; IBM Corp).

\section{Results}

\section{Demographics}

A total of 188 patients met the inclusion criteria and were included in the analysis (Fig. 1). There were 53\% male hips $(100 / 188)$ included. The mean patient age was $61.2 \pm 10.2$ (30.0-83.0) years, the mean body mass index (BMI) was $27.1 \pm 4.6(17.1-38.6) \mathrm{kg} / \mathrm{m}^{2}$, and the mean ASA score was $2.0 \pm 0.6(1.0-3.0)$ points. The percentages of negative prognostic factors (diabetes, depression, chronic pain syndrome) did not differ significantly among the groups. The proportions of patients who underwent arthroplasty of the contralateral hip or knee joint did not differ significantly. Ninety-eight THAs were performed on the right side, and

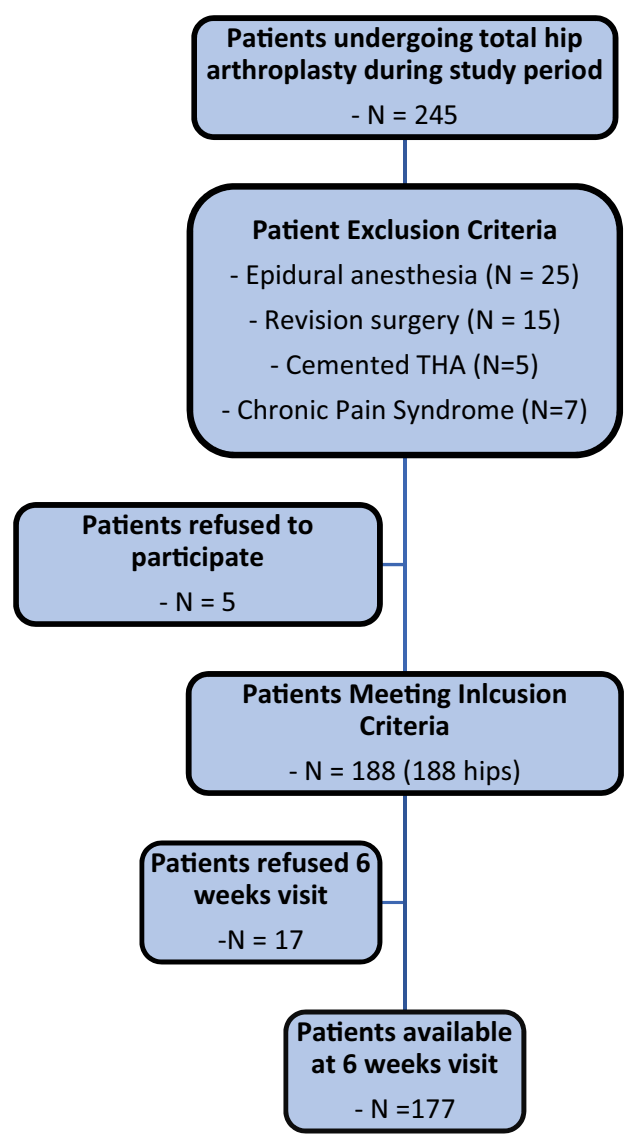

Fig. 1 Flowchart illustrating the number of patients excluded from the study, lost to follow-up, and those who met the inclusion criteria
90 were performed on the left side. A total of 132 patients were discharged on the fourth postoperative day, and 56 were discharged on the fifth postoperative day. A significant difference was found among the three groups regarding the respective operation time ( $p<0.006$, ANOVA). In the post hoc pairwise comparisons (Bonferroni correction), we found statistically significant differences between groups 1 and 2 $(p=0.003)$ and groups 2 and $3(p=0.0019)$. No significant differences were found when comparing groups 1 and 3 $(p=0.356)$. Table 1 shows the data for each approach group.

\section{Postoperative mobilization}

A total of 185 (98\%) patients could be mobilized on the day of surgery. In $3(2 \%)$ patients, the first mobilization was only on the first postoperative day. The reason for the delay in these patients was increased nausea and circulatory weakness. The walking distance within the individual groups increased significantly from day to day $(p<0.001)$ (Fig. 2). Comparing the walking distance between the different groups, no significant differences were found for the first 2 postoperative days, with patients operated on using the lateral approach tending to walk a shorter distance. There was a significant difference on the third postoperative day. Patients in groups 1 and 3 had a significantly longer walking distance than those in group 2 (group1 vs. group 2, $p=0.02$; group 2 vs. group $3, p=0.03)$. There was no difference between groups 1 and $3(p=0.832)$.

\section{Pain Scores}

Pain was assessed preoperatively, as well as daily during the hospital stay and for 6 weeks of follow-up at rest and during motion. The pain level decreased significantly over time in each group $(p<0.001)$. However, there were differences regarding the different points of time. DAA patients reported significantly less pain at rest at 6 weeks than in the lateral and posterior approach groups (group 1 vs. group 2, $p=0.004$; group 1 vs. group $3, p=0.007$, group 2 vs. group $3, p=0.371$ ). There were no differences among the groups during the inpatient stay for the pain level at rest.

DAA and posterior approach patients reported significantly less pain during motion on the third postoperative day and at 6 weeks than the lateral approach group (third postoperative day: group 1 vs. group $2, p=0.011$; group 1 vs. group $3, p=0.563$, group 2 vs. group $3, p=0.04 ; 6$ weeks: group 1 vs. group $2, p=0.001$; group 1 vs. group $3, p=0.300$, group 2 vs. group $3, p=0.005$ ) (Table 2 ).

\section{Analysis of pre-versus postoperative mHHS}

The data of 177 patients were available for evaluation of the mHHS at the 6-week follow-up. The mHHS 
Table 1 Patient demographic data according to the different approaches

\begin{tabular}{|c|c|c|c|c|}
\hline Approach & DAA & Lateral & Posterior & $p$ value \\
\hline Total no. of patients & 88 & 26 & 74 & \\
\hline \multicolumn{5}{|l|}{ Laterality, $n(\%)$} \\
\hline Right & $48(55)$ & $14(54)$ & $36(49)$ & 0.832 \\
\hline Left & $40(45)$ & $12(46)$ & $38(51)$ & 0.793 \\
\hline \multicolumn{5}{|l|}{ Sex, $n(\%)$} \\
\hline Male & $42(48)$ & $14(54)$ & $44(59)$ & 0.421 \\
\hline Female & $46(52)$ & $12(46)$ & $30(41)$ & 0.391 \\
\hline Age, y & $61.9 \pm 9.8(30.0-81.0)$ & $60.3 \pm 12.3(32.0-83.0)$ & $60.7 \pm 9.7(31.0-81.0)$ & 0.798 \\
\hline Body mass index, $\mathrm{kg} / \mathrm{m}^{2}$ & $26.9 \pm 4.1(19.2-38.0)$ & $27.0 \pm 4.8(17.1-36.3)$ & $27.2 \pm 4.9(20.8-38.6)$ & 0.832 \\
\hline ASA Score & $2.0 \pm 0.5(1.0-3.0)$ & $1.9 \pm 0.6(1.0-3.0)$ & $1.9 \pm 0.5(1.0-3.0)$ & 0.865 \\
\hline \multicolumn{5}{|c|}{ Negative prognostic comorbidities $n(\%)$} \\
\hline Diabetes & $6(7)$ & $2(8)$ & $5(7)$ & 0.986 \\
\hline Depression & $4(5)$ & $1(4)$ & $4(5)$ & 0.940 \\
\hline Chronic Pain Syndrome & $2(2)$ & $0(0)$ & $2(3)$ & 0.708 \\
\hline \multicolumn{5}{|l|}{ Previous arthroplasties $n(\%)$} \\
\hline THA of the contralateral side & $12(14)$ & $4(15)$ & $12(6)$ & 0.897 \\
\hline TKA & $7(8)$ & $2(8)$ & $5(7)$ & 0.958 \\
\hline \multicolumn{5}{|c|}{ Preoperative pain medication $n(\%)$} \\
\hline NSAID & $66(75)$ & $19(73)$ & $53(72)$ & 0.134 \\
\hline Opioids & $8(9)$ & $2(8)$ & $5(7)$ & 0.860 \\
\hline \multicolumn{5}{|l|}{ Use of walking aids $n(\%)$} \\
\hline One walking cane & $1(1)$ & $0(0)$ & $1(1)$ & 0.843 \\
\hline Two crutches & $0(0)$ & $0(0)$ & $1(1)$ & 0.461 \\
\hline
\end{tabular}

Values are shown as $n(\%)$, respectively as the mean \pm SD (range)

ASA American Society of Anesthesiologists; DAA direct anterior approach; NSAID nonsteroidal anti-inflammatory drugs

Fig. 2 Presentation of the walking distances for the individual groups; DAA, direct anterior approach

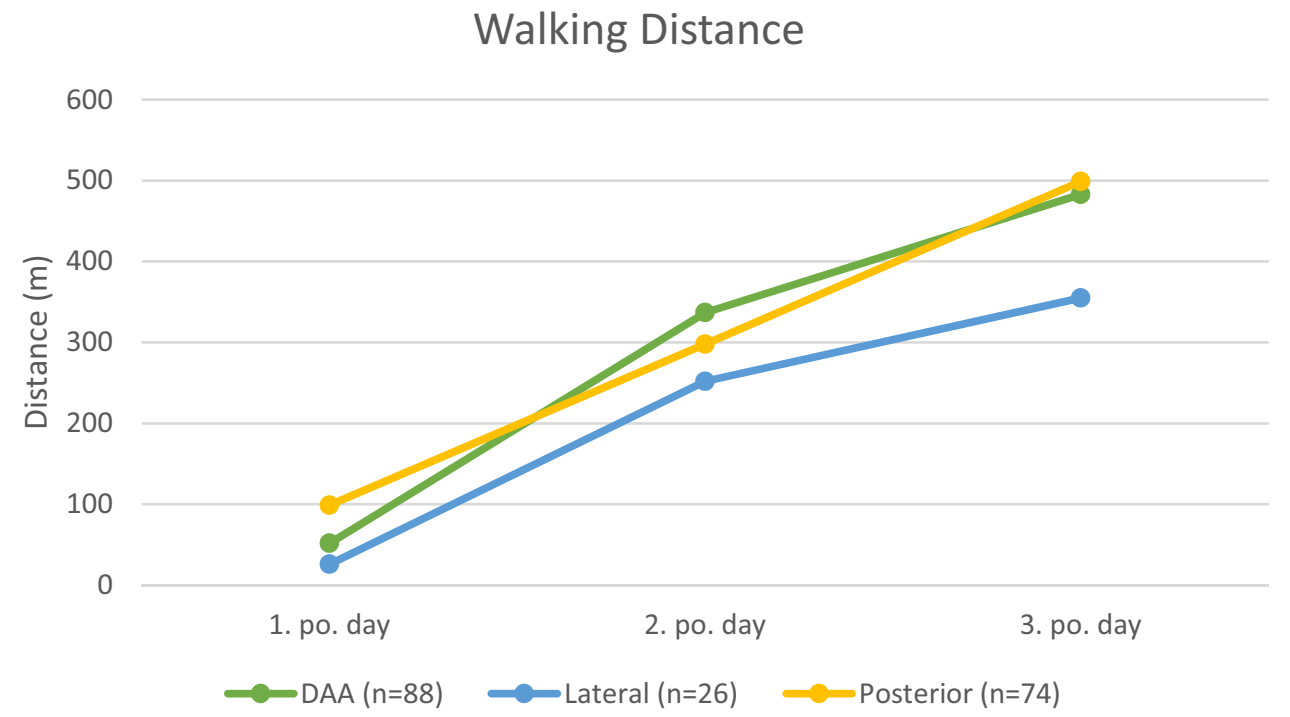

demonstrated statistically significant improvement within each group: group 1: preoperative 34.8 44.8 (20.0-50.0) vs. follow-up $85.5 \pm 14.6$ (41.8-100.0), $p<0.00001$; group 2: preoperative $32.4 \pm 4.7(20.0-41.0) \mathrm{vs}$. follow-up $76.2 \pm 15.8$ (37.4-97.9), $p<0.00001$; group 3 : preoperative $34.8 \pm 4.9(24.0-45.0)$ vs. follow-up $85.4 \pm 13.5$ (41.8-100.0), $p<0.00001)$ (Fig. 3)

A significant difference was found among the three groups regarding the respective change in the $\mathrm{mHHS}$ value $(p<0.033$, ANOVA). In the post hoc pairwise comparisons 
Table 2 Pain levels within the approach groups at the different time points

\begin{tabular}{llllll}
\hline Approach & Preoperative & First postoperative day & Second postoperative day & Third postoperative day & 6 weeks postoperative \\
\hline \multirow{2}{*}{ DAA } & VAS pain for rest & & & & \\
Lateral & $4.7 \pm 2.8(0.0-10.0)$ & $1.3 \pm 1.5(0.0-8.0)$ & $1.1 \pm 1.3(0.0-6.0)$ & $0.8 \pm 1.1(0.0-6.0)$ & $0.2 \pm 0.5(0.0-2.0)^{\mathrm{a}}$ \\
Posterior & $4.5 \pm 3.1(0.0-10.0)$ & $1.8 \pm 1.6(0.0-6.0)$ & $1.3 \pm 1.5(0.0-6.0)$ & $1.1 \pm 1.5(0.0-6.0)$ & $0.6 \pm 0.8(0.0-3.0)^{\mathrm{a}}$ \\
& VAS pain for motion & & $1.2 \pm 1.1(0.0-4.0)$ & $1.0 \pm 1.1(0.0-4.0)$ & $0.6 \pm 1.1(0.0-4.0)^{\mathrm{a}}$ \\
DAA & $7.3 \pm 2.0(2.0-10.0)$ & $4.8 \pm 2.2(0.0-10.0)^{\mathrm{b}}$ & $3.8 \pm 2.1(0.0-9.0)$ & $3.0 \pm 2.0(0.0-8.0)^{\mathrm{c}}$ & $1.1 \pm 1.1(0.0-4.0)^{\mathrm{c}}$ \\
Lateral & $7.4 \pm 2.0(3.0-10.0)$ & $5.9 \pm 2.4(1.0-10.0)^{\mathrm{b}}$ & $4.6 \pm 2.9(0.0-10.0)$ & $4.4 \pm 2.7(0.0-10.0)^{\mathrm{c}}$ & $2.1 \pm 1.4(0.0-6.0)^{\mathrm{c}}$ \\
Posterior & $6.5 \pm 2.2(0.0-10.0)$ & $5.2 \pm 2.2(1.0-10.0)$ & $4.0 \pm 1.9(1.0-8.0)$ & $3.1 \pm 1.8(0.0-8.0)^{\mathrm{c}}$ & $1.3 \pm 1.3(0.0-6.0)^{\mathrm{c}}$ \\
\hline
\end{tabular}

Presentation of the pain level based on VAS pain for the individual approach groups at the different points in time

$D A A$ direct anterior approach; VAS visual analog scale

${ }^{a}$ DAA patients reported significantly less pain at rest at 6 weeks control than the lateral and posterior approach groups

${ }^{b}$ DAA patients reported significantly less pain during motion at the 1st postoperative day than the lateral approach group

${ }^{c}$ DAA and posterior approach patients reported significantly less pain at motion at the third postoperative day and at 6 weeks than the lateral approach group

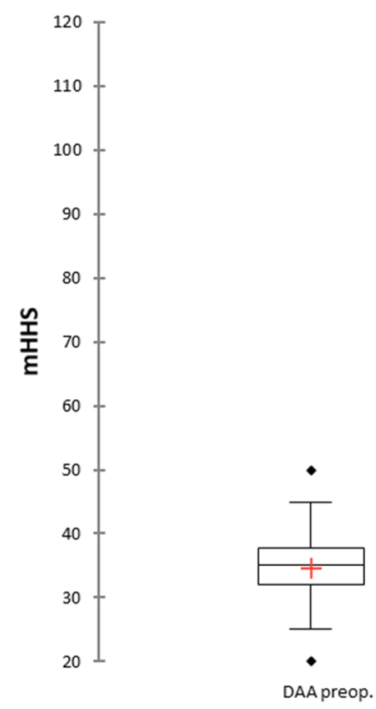

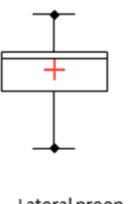
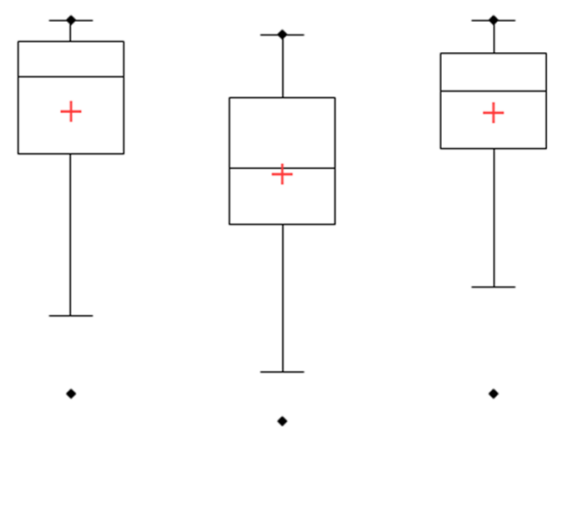

Lateral preop.

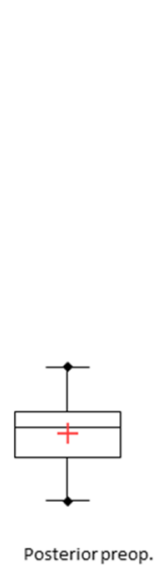

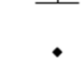

Lateral 6 weeks postop.

Fig. 3 Presentation of the mHHS values for the respective approach groups; comparison of preoperative to 6-week follow-up. $m H H S$ modified Harris Hip Score

(Bonferroni correction), we found statistically significant differences between groups 1 and $2(p=0.006)$ and groups 2 and $3(p=0.019)$. No significant differences were found when comparing groups 1 and $3(p=0.656)$. The lateral approach group showed significantly less improvement (Fig. 4).

\section{Discussion}

This study analyzed the influence of the surgical approach on perioperative pain and short-term rehabilitation after THA implantation. The main finding was that DAA patients reported the least pain on the third postoperative day and at the 6-week follow-up. In addition, patients treated with a DAA and posterior approach achieved a significantly longer walking distance and thus faster mobilization during the inpatient stay. Patients operated on with the lateral approach achieved the least improvement in the mHHS after 6 weeks. This is significant because, to the best of our knowledge, this is the first study comparing the perioperative effect of three different surgical approaches for THA.

In modern endoprosthetics, the aim is to damage as little soft tissue as possible, which enables faster mobilization and rehabilitation. Most of our patients could already be mobilized on the day of surgery. For the first 2 postoperative 


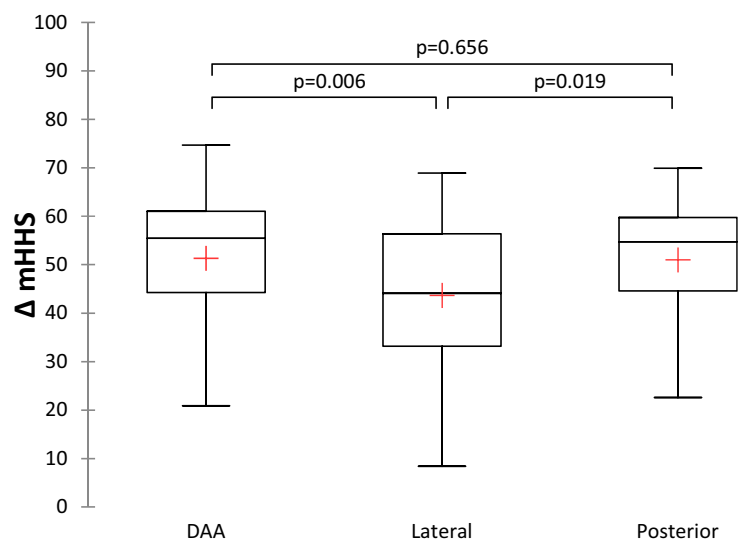

Fig. 4 Presentation of the $\Delta$ in the mHHS for each group. $D A A$ direct anterior approach; $m H H S$ modified Harris Hips Score

days, there were no significant differences in the walking distance achieved in the group comparison. However, patients with the DAA and posterior approach had a significantly longer walking distance on the third postoperative day than those with the lateral approach. Thus, patients who underwent surgery with a minimally invasive approach achieved a longer walking distance by the discharge day. These results are in contrast with Barrett et al.'s study, which compared the anterior and posterior approaches. The DAA subjects walked further postoperatively on the day of surgery and the first and second days postoperatively [25]. The reason for these different results may be due to the surgical technique used for the posterior approach. In our study, the piriformis tendon was not detached and preserved, whereas in Barrett's study, this was not explicitly mentioned. Martusiewicz et al. demonstrated the same walking distance on the discharge day for the anterior and posterior approaches [26].

Another factor influencing perioperative mobilization is pain. Patients operated on with the DAA had significantly less pain at rest than the lateral and posterior approach patients at the 6-week follow-up. Regarding pain during motion, patients with the DAA and posterior approaches had significantly less pain on the third postoperative day and at the 6-week follow-up than patients with the lateral approach. The abductor complex is a pain generator following the direct lateral approach and this may explain differences in early pain perception between the groups [27]. A recent study by Seah et al. investigated postoperative pain and subsequent opioid consumption between three surgical approaches in patients undergoing primary elective THA [16]. In their study, DAA was associated with lower daily opioid usage and pain scores after elective THA in the early postoperative period. Our results confirm these findings, whereby the posterior approach also showed significantly less pain during motion at the third postoperative day and at 6 weeks than the lateral approach.
However, our results are in contrast with other studies, that reported lower pain severity for DAA compared to the posterior approach $[9,11,12]$. Nevertheless, the authors could not identify any reason for this and called it speculative [9].

Certain comorbidities have been identified in the literature that can negatively affect postoperative outcomes, especially pain. These factors include diabetes, depression, and chronic pain syndrome [20-23]. In our cohort, there were no significant differences in the distributions of these factors across the groups. Therefore, we assume that these factors did not influence the outcome of the present study. Another possible factor that may influence the perioperative outcome is the duration of surgery. In our cohort, the operation time was significantly longer in the lateral approach group than in the DAA and posterior approach groups. However, it has recently been shown that the duration of surgery has no influence on the postoperative outcome [28], so we do not postulate it had a significant effect on the study outcome. The average operation times in our study were below the reported average operation time for a THA of 93 min [29].

Our study surveyed the mHHS as a PROM. We showed that the lateral approach resulted in a significantly lower improvement than the DAA or the posterior approach. The DAA and posterior approach showed similar improvements at the 6-week follow-up. These findings are consistent with other studies that found less improvements in PROMs for the lateral approach at short-term follow-up $[17,30]$. These studies also showed no difference in the short-term results in the PROMS when comparing the DAA with the posterior approach [11, 17].

\section{Strengths and limitations}

One strength of this study is its prospective nature and the inclusion of a consecutive series of patients who received THA. Another advantage is that each surgery was performed by high-volume surgeons, each of whom had a high level of experience in THA.

Our study is not free of limitations. First, we only report a short follow-up period of 6 weeks, so the consistency of the results cannot be assessed. Follow-up studies must, therefore, determine whether the differences found will have persisted. Second, the procedures were carried out by several surgeons, so that individual influences cannot be excluded, and therefore, a surgeon-related bias may exist. However, surgeon selection bias was likely not a factor, as each surgeon performed only the assigned approach during the study period. The lateral approach was performed by only one surgeon, so this group had a small size, which may have influenced the results. 


\section{Conclusion}

This study analyzed perioperative pain progression and short-term rehabilitation after THA according to the different surgical approaches. Direct anterior and posterior approaches have shown comparable improvements in pain, walking distance, and mHHS. Whether this effect persists over a longer period of time must be clarified in future studies.

Funding Open Access funding enabled and organized by Projekt DEAL. Funding was not applicable.

\section{Declarations}

Conflict of interest The authors report no conflict of interest.

Open Access This article is licensed under a Creative Commons Attribution 4.0 International License, which permits use, sharing, adaptation, distribution and reproduction in any medium or format, as long as you give appropriate credit to the original author(s) and the source, provide a link to the Creative Commons licence, and indicate if changes were made. The images or other third party material in this article are included in the article's Creative Commons licence, unless indicated otherwise in a credit line to the material. If material is not included in the article's Creative Commons licence and your intended use is not permitted by statutory regulation or exceeds the permitted use, you will need to obtain permission directly from the copyright holder. To view a copy of this licence, visit http://creativecommons.org/licenses/by/4.0/.

\section{References}

1. Lavernia CJ, Alcerro JC (2011) Quality of life and cost-effectiveness 1 year after total hip arthroplasty. J Arthroplasty 26(5):705709. https://doi.org/10.1016/j.arth.2010.07.026

2. Ethgen $\mathrm{O}$ et al (2004) Health-related quality of life in total hip and total knee arthroplasty a qualitative and systematic review of the literature. J Bone Joint Surg Am 86(5):963-974

3. Pennington MW, Grieve R, van der Meulen JH (2015) Lifetime cost effectiveness of different brands of prosthesis used for total hip arthroplasty. Bone Joint J 97B(6):762-770

4. Dailiana ZH, Papakostidou I, Varitimidis S et al (2015) Patientreported quality of life after primary major joint arthroplasty: a prospective comparison of hip and knee arthroplasty. BMC Musculoskelet Disord 16(1):646

5. Graves SC, Dropkin BM, Keeney BJ et al (2016) Does surgical approach affect patient-reported function after primary THA? Clin Orthop Relat Res 474(4):971-981

6. Müller M, Tohtz S, Dewey M et al (2011) Age-related appearance of muscle trauma in primary total hip arthroplasty and the benefit of a minimally invasive approach for patients older than 70 years. Int Orthopaed (SICOT) 35(2):165-171

7. Wang T, Shao L, Xu W et al (2019) Comparison of morphological changes of gluteus medius and abductor strength for total hip arthroplasty via posterior and modified direct lateral approaches. Int Orthopaed (SICOT) 43(11):2467-2475

8. Talia AJ, Coetzee C, Tirosh O et al (2018) Comparison of outcome measures and complication rates following three different approaches for primary total hip arthroplasty: a pragmatic randomised controlled trial. Trials 19(1):1-9

9. Miller LE, Gondusky JS, Bhattacharyya S et al (2018) Does surgical approach affect outcomes in total hip arthroplasty through 90 days of follow-up?: A systematic review with meta-analysis. J Arthroplasty 33(4):1296-1302

10. Higgins BT, Barlow DR, Heagerty NE et al (2015) Anterior vs. posterior approach for total hip arthroplasty, a systematic review and meta-analysis. J Arthroplasty 30(3):419-434

11. Wang Z, Hou J-z, Wu C-h et al (2018) A systematic review and meta-analysis of direct anterior approach versus posterior approach in total hip arthroplasty. J Orthop Surg Res 13(1):335

12. Miller LE, Kamath AF, Boettner F et al (2018) In-hospital outcomes with anterior versus posterior approaches in total hip arthroplasty: meta-analysis of randomized controlled trials. JPR 11:1327-1334

13. Kobayashi H, Homma Y, Baba T et al (2016) Surgeons changing the approach for total hip arthroplasty from posterior to direct anterior with fluoroscopy should consider potential excessive cup anteversion and flexion implantation of the stem in their early experience. Int Orthopaed (SICOT) 40(9):1813-1819

14. Radoicic D, Zec V, Elassuity WI et al (2018) Patient's perspective on direct anterior versus posterior approach total hip arthroplasty. Int Orthopaed (SICOT) 42(12):2771-2775

15. Parvizi J, Rasouli MR, Jaberi M et al (2013) Does the surgical approach in one stage bilateral total hip arthroplasty affect blood loss? Int Orthopaed (SICOT) 37(12):2357-2362

16. Seah S, Quinn M, Tirosh O et al (2019) Postoperative opioid consumption after total hip arthroplasty: a comparison of three surgical approaches. J Arthroplasty 34(11):2676-2680

17. Peters RM, van Beers LWAH, van Steenbergen LN et al (2018) Similar superior patient-reported outcome measures for anterior and posterolateral approaches after total hip arthroplasty. J Arthroplasty 33(6): 1786-1793

18. Harris WH (1969) Traumatic arthritis of the hip after dislocation and acetabular fractures: treatment by mold arthroplasty. An endresult study using a new method of result evaluation. J Bone Joint Surg Am 51(4):737-755

19. Byrd JWT (2003) Hip arthroscopy: patient assessment and indications. Instr Course Lect 52:711-719

20. Davis JA, Robinson RL, Le TK et al (2011) Incidence and impact of pain conditions and comorbid illnesses. J Pain Res 4:331-345

21. Alenazi AM, Obaidat SM, Alshehri MM et al (2020) Type 2 diabetes affects joint pain severity in people with localized osteoarthritis: a retrospective study. Pain Med 21(5):1025-1031

22. Lindner M, Nosseir O, Keller-Pliessnig A et al (2018) Psychosocial predictors for outcome after total joint arthroplasty: A prospective comparison of hip and knee arthroplasty. BMC Musculoskelet Disord 19(1):159

23. Schwartz FH, Lange J (2017) Factors that affect outcome following total joint arthroplasty: a review of the recent literature. Curr Rev Musculoskelet Med 10(3):346-355

24. Faul F, Erdfelder E, Buchner A et al (2009) Statistical power analyses using $\mathrm{G}^{*}$ Power 3.1: tests for correlation and regression analyses. Behav Res Methods 41(4):1149-1160

25. Barrett WP, Turner SE, Leopold JP (2013) Prospective randomized study of direct anterior vs postero-lateral approach for total hip arthroplasty. J Arthroplasty 28(9):1634-1638

26. Martusiewicz A, Delagrammaticas D, Harold RE et al (2019) Anterior versus posterior approach total hip arthroplasty: Patientreported and functional outcomes in the early postoperative period. Hip Int 14:112070001988141

27. Lachiewicz PF (2011) Abductor tendon tears of the hip: evaluation and management. J Am Acad Orthop Surg 19(7):385-391 
28. Weber M, Benditz A, Woerner M et al (2017) Trainee surgeons affect operative time but not outcome in minimally invasive total hip arthroplasty. Sci Rep 7(1):6152

29. Cantrell WA, Samuel LT, Sultan AA et al (2019) Operative times have remained stable for total hip arthroplasty for 15 years: systematic review of 630,675 procedures. JB JS Open Access 4(4): $\mathrm{e} 0047$

30. Restrepo C, Parvizi J, Pour AE et al (2010) Prospective randomized study of two surgical approaches for total hip arthroplasty. J Arthroplasty 25(5):671-679.e1
Publisher's Note Springer Nature remains neutral with regard to jurisdictional claims in published maps and institutional affiliations. 